\title{
Énumération et structuration discursive
}

\author{
Rebeyrolle, Josette \& Péry-Woodley, Marie-Paule \\ CLLE-ERSS (CNRS \& Université de Toulouse-Le Mirail) \\ \{josette.rebeyrolle, pery\}@univ-tlse2.fr
}

\section{Introduction}

Comme point de départ de l'examen de l'énumération en discours, nous proposons deux exemples : dans le premier, les cinq items se suivent au fil du texte, ils sont annoncés et ordonnés par des marqueurs lexicaux (disposition horizontale); dans le second, les items sont agencés verticalement (alinéa, retrait) et marqués par des puces ${ }^{1}$.

(1) Les facteurs essentiels sont ici les suivants. En premier lieu, la guerre a en général pour les acteurs locaux un intérêt politique de premier ordre, souvent bien plus important que celui des Etats-Unis. Leur tolérance à la souffrance est donc plus grande. En deuxième lieu, en dépit de leur taille réduite, ces acteurs supplantent d'ordinaire les Etats-Unis dans une ressource précise : le nombre d'hommes en âge de combattre. Même s'il n'est plus l'élément déterminant de la guerre terrestre, il reste un facteur critique, notamment en ville, dans la jungle ou en montagne. Troisièmement, les " locaux " disposent en général d'un avantage : ils jouent à domicile. Si les Etats-Unis ont constitué au fil des décennies la mémoire institutionnelle qui leur permet de maintenir leur maîtrise des espaces, les acteurs locaux ont fait un travail similaire sur leur propre pays. Ils connaissent intimement le terrain et la météo, et ont mis au point, sur des décennies, voire des siècles, des tactiques et des stratégies adaptées à leurs milieux. Quatrièmement, nombre des chefs militaires de ces Etats ou entités ont été formés dans le monde développé - pendant la guerre froide, la formation militaire fut souvent utilisée comme instrument d'influence politique. Ils ont appris les tactiques en vigueur en Occident, comme l'usage des armes occidentales, et les meilleurs d'entre eux peuvent tourner ces connaissances contre les EtatsUnis. Certains rapports montrent d'ailleurs que les adversaires des Etats-Unis ont échangé leurs expériences. Cinquièmement, l'arsenal nécessaire au combat rapproché, à terre, dans les airs à basse altitude ou dans les eaux territoriales est beaucoup moins coûteux que les armements nécessaires à la guerre dans les " espaces communs ". En outre, la diffusion des capacités économiques et technologiques civiles trouve son parallèle dans le domaine militaire: de nouveaux fabricants apparaissent, cherchant des débouchés à l'export, et l'arsenal pour le combat rapproché connaît un perfectionnement constant. Tous ces facteurs se renforcent et contribuent à créer une "zone contestée". (Géopolitique)

(2) En substance, il est reproché à l'analogie :

- de ne pas permettre de bonnes prédictions sur les formes possibles et impossibles,

- d'être insuffisamment contrainte,

- de ne pas permettre de généralisation,

- d'avoir contre elle des évidences psychologiques,

- de ne pas produire de résultats différents de ceux que produisent les modèles statistiques non analogiques.

Cependant, à l'issue de l'examen de chacune de ces critiques, rien de décisif ne se dégage. (Linguistique)

À travers la diversité des marques - on peut voir (1) et (2) comme se situant aux deux pôles d'un continuum qui irait d'une formulation «discursive » à une formulation « visuelle » - il s'agit pour nous de la même structure, avec amorce, items clairement identifiables et clôture. Dans cet article, après avoir précisé notre approche et son ancrage (section 2), notre premier objectif sera précisément d'aborder la diversité des réalisations des structures énumératives (section 3), tout en dégageant clairement ce qui en fait l'unité : la mise en parallèle des items, l'expression (ou l'inférabilité) du critère interprétatif qui soustend cette mise en parallèle (en (1), ce sont des facteurs, en (2), des critiques). Nous évoquerons aussi la diversité des contextes où elles s'insèrent et des rôles discursifs auxquels elles se prêtent ainsi que les premiers résultats concernant les corrélations entre types de réalisation et fonction. La dernière section sera consacrée aux «marges» de la structure - l'amorce, qui la lie au texte amont et annonce l'énumération, et la clôture, segment final qui fait le lien avec le texte aval-pour mieux mettre en lumière la nécessité de traiter la structure dans son ensemble comme un tout fonctionnel.

\section{Listes, séries linéaires, structures énumératives}

Notre propos est ici d'examiner l'énumération comme procédé de structuration du discours. Si la liste a suscité l'intérêt des stylisticiens (cf. Milcent-Lawson et al., 2013), c'est surtout pour son aptitude à sidérer, à produire le vertige. À l'autre extrême d'un continuum disciplinaire imaginaire, les chercheurs en traitement automatique des langues ont pu s'intéresser aux énumérations comme porteuses de connaissances sur des mondes à modéliser, y cherchant des relations de type taxinomiques. Du côté de la 
linguistique, le caractère insistant de certains marqueurs d'items, leur fonctionnement en système, ont intrigué des linguistes et suscité un certain nombre de travaux : autour de la notion de «sequencer » pour les travaux anglophones (cf. Hempel et Degand, 2008), de «Marqueur d'Intégration Linéaire » $\left(\mathrm{MIL}^{2}\right.$ ) pour les travaux francophones ${ }^{3}$. Des études se sont appuyées sur ce marquage pour localiser et extraire les séries d'items dans des textes diversifiés et ont commencé à s'interroger sur le fonctionnement de ces « séries linéaires » en discours (Jackiewicz, 2005). C'est pleinement sous cet angle du fonctionnement discursif que quelques auteurs se sont penchés sur les interactions possibles entre énumération et relations de discours : Luc et al. (2000) considèrent qu'elles appartiennent à des modes d'organisation distincts qu'il s'agit d'articuler, ce qu'ils font à la lumière du modèle de l'architecture textuelle d'une part et de la Rhetorical Structure Theory de l'autre; Bras et al. (2008), Vergez-Couret et al. (2012) créent au contraire pour en rendre compte une relation d'énumération et intègrent ainsi l'énumération dans la Segmented Discourse Representation Theory, une approche de la cohérence qui repose sur la construction de segments complexes reliés par des relations de discours. La ressource ANNODIS ${ }^{4}$ (corpus de français écrit enrichi d'annotations discursives), où les structures énumératives ont fait l'objet d'une annotation systématique dans un corpus diversifié, a permis d'en montrer la fréquence et la diversité des réalisations (Ho-Dac et al., 2012 ; Ho-Dac et Péry-Woodley, 2014 - ce volume).

On verra que notre approche se fonde - à des titres différents - sur plusieurs des travaux rapidement parcourus ci-dessus. Nous tentons dans ce qui suit de la caractériser à la fois dans ses ancrages et dans ses objectifs. À la suite des travaux sur les MIL et les sequencers, nous visons l'identification des marqueurs de structures énumératives mais d'une manière qui s'en distingue toutefois et nécessite d'être précisée. Parmi ces précisions, les deux premières ont trait à notre ancrage dans le modèle d'architecture textuelle.

Premièrement, et c'est ce que vise à montrer l'exemple (2), nous ne privilégions pas les marqueurs lexicaux et prenons pleinement en considération la typo-disposition et la ponctuation ; dans un même ordre d'idée, nous considérons qu'on ne peut associer un rôle discursif à un élément lexical sans tenir compte de son inscription spécifique dans le texte - c'est-à-dire sa fonction syntaxique, sa position textuelle, son environnement typo-dispositionnel.

Deuxièmement, nous envisageons les structures énumératives comme un procédé textuel de base (une sorte de «brique textuelle ») que l'on peut décrire comme le fait d'agencer du texte de façon que le lecteur soit amené à prendre conscience de cet agencement. Dans les termes de Luc et al. (2000), cet agencement constitue un acte textuel: le scripteur dispose du texte de manière que le lecteur reconnaisse à la fois la disposition et l'intention qui lui a présidé. L'intention est que soit reconnue l'identité des éléments énumérés (item/idem) en relation à un critère spécifique. Ce critère, explicite ou inférable, sera appelé critère de co-énumérabilité.

Il s'ensuit que l'énumération nous intéresse en tant qu'agencement textuel. Nous défendrons l'idée qu'un tel agencement réalise une stratégie de discours, et constitue par conséquent une structure discursive irréductible à une série d'items signalés par des marqueurs linguistiques spécifiques. Notons par ailleurs que là où les travaux sur les marqueurs ont cherché à y voir une manifestation principalement métadiscursive, sans apport sur le plan du contenu propositionnel, nous mettons au contraire l'accent sur le double rôle des structures énumératives, qui tout à la fois organisent des portions de texte et réalisent des contenus. Nous nous rapprochons ce faisant de la linguistique systémique fonctionnelle, selon laquelle les métafonctions textuelle et idéationnelle se réalisent conjointement (cf. Halliday, 1977). S'il est possible d'interpréter certains types de marqueurs, pris isolément, comme spécialisés dans l'organisation du texte, la prise en compte de la signalisation de la structure dans son ensemble donne à voir qu'elle met en œuvre deux procédés indissociables : la segmentation/mise en séquence des items et l'expression du critère de co-énumérabilité. Il arrive qu'un type de marqueur remplisse les deux rôles à la fois : c'est le cas des titres par exemple, ou des adverbiaux en position initiale de paragraphe. Dans d'autres cas, les rôles se répartissent sur des marques différentes : puces et numérotation signalent la segmentation/mise en séquence, en association avec l'expression lexicale du critère de co-énumérabilité. Les exemples traités dans la section suivante vont illustrer ces différents cas de figure. 


\section{La structure énumérative à travers la diversité des formes}

Pour illustrer la variété des réalisations de la structure, nous exploitons la ressource ANNODIs, et plus précisément les résultats de l'annotation manuelle des structures énumératives. Nous ne remettons pas en question la définition des structures qui a présidé à l'annotation mais nous utilisons ce vaste ensemble de données pour examiner la diversité des réalisations avant de décrire les fonctions discursives de ces structures. Au moment de cet examen, nous nous autorisons cependant un regard critique.

Tous nos exemples sont donc issus du corpus ANNODIS qui est, rappelons-le, composé de textes diversifiés sur le plan du genre discursif et du domaine et s'organise en trois parties : 1) des articles de recherche dans le domaine de la linguistique, 2) des rapports et articles dans le domaine de la géopolitique et 3) des articles issus de Wikipédia ${ }^{5}$.

Étant donné le nombre de structures annotées mises à disposition ${ }^{6}$, on aurait pu être tenté de multiplier les «jolis» exemples. Nous avons fait le choix de nous contenter des quatre suivants parce que nous voudrions mettre l'accent sur deux propriétés fondamentales de ces structures. Nos exemples serviront d'une part à montrer comment la signalisation des items est loin d'être réservée aux MIL (cf. ci-dessus l'exemple (1)). Peuvent également jouer ce rôle - tout en s'associant éventuellement à des MIL - d'autres marqueurs lexicaux (exemples 3, 5 et 6), mais aussi la typo-disposition (exemple (2) ci-dessus et exemple (4) ici), ou le parallélisme syntaxique (3, 4, 5 et 6$)$. Et d'autre part ils illustreront l'empan textuel des agencements que les structures énumératives permettent: de quelques lignes à des sections entières sur une ou plusieurs pages (exemple (4)).

Dans l'exemple (3) ci-dessous, l'énumération apparaît nettement puisqu'elle est doublement signalée : à la fois par la série de trois syntagmes prépositionnels juxtaposés et étroitement parallèles - chacun étant introduit par la préposition par dans un syntagme détaché en tête de phrase - et par la série de MIL temporels (tout d'abord, ensuite, enfin). Le rattachement de ces syntagmes prépositionnels exige du lecteur au terme de la séquence qu'il établisse une relation entre un cap a été franchi et la série d'items qui suit.

(3) Pourtant, les attaques du 11 septembre et la psychose entretenue par les découvertes d'enveloppes contenant du bacille du charbon donnent le sentiment qu'un cap a été franchi. Par le nombre très élevé de victimes, tout d'abord, qui rend dérisoire le qualificatif d'attentat et incite à évoquer un acte de guerre, même si l'absence d'ennemi identifié ne rend pas ce terme vraiment satisfaisant. Par la nature des cibles, ensuite, symboles de la puissance militaire et économique des États-Unis, qui donnent la mesure des intentions et de l'idéologie destructrice qui animent les terroristes. Par le mode opératoire choisi, enfin, qui accentue ce sentiment de grande vulnérabilité : en détournant des avions de ligne intérieure ou - s'il s'avère que les coupables sont les mêmes - en utilisant le système postal comme vecteur de leurs attaques bactériologiques, les terroristes ont détourné l'utilisation des fondements de la société américaine que sont la libre circulation, les échanges et la communication. (Géopolitique)

L'exemple (4) relève de la même stratégie de discours et cela même si la structure organise ici le contenu de l'ensemble d'une section dans laquelle ce sont les titres qui marquent les items. Cet exemple illustre la fonction de structuration textuelle de la structure et plus précisément sa capacité à s'articuler avec la structure de document ${ }^{7}$ pour englober des sections entières quand, comme ici, le titre exprime le critère de co-énumérabilité et que le critère qu'elle énonce s'étend sur des items qui prennent la forme de sections titrées. Les titres d'un même niveau mettent ainsi en parallèle les paragraphes qu'ils chapeautent, constituant autant d'items de l'énumération. Les items en (4) sont clairement signalés comme coénumérables : par la mise en forme matérielle - chacun des items fait l'objet d'un paragraphe distinct ; par le parallélisme syntaxique des syntagmes placés en titre ; par l'annonce des items (qui a ici la forme de ce que Porhiel (2007) appelle une énumération à deux temps, cf. section 4.1). Lorsque, comme ici, la structure du document porte la structure textuelle, le marquage visuel joue un rôle important dans l'interprétation énumérative du texte notamment parce qu'il force le parallélisme. Les trois principes de la sélection naturelle forment ainsi chacun un item. L'expression linguistique des co-items repose sur une reprise du nom principes dont la première mention se trouve dans le titre - où ce nom au pluriel rend saillant le critère qui rassemble les items de l'énumération. Ce type de répétition instaure une forte cohésion lexicale.

(4) II. Principes de la sélection naturelle

La théorie de la sélection naturelle telle qu'elle a été initialement décrite par Charles Darwin, repose sur trois principes : 


\begin{abstract}
- le principe de variation
- le principe d'adaptation

- le principe d'hérédité

II.1. Principe $1:$ Les individus diffèrent les uns des autres

En général, dans une population d'individus d'une même espèce, il existe des différences plus ou moins importantes entre ces individus. En biologie, on appelle caractère, tout ce qui est visible et peut varier d'un individu a a 'autre. On dit qu'il existe plusieurs traits pour un même caractère. Par exemple, chez l'être humain, la couleur de la peau, la couleur des yeux sont des caractères pour lesquels il
existe de multiples variations ou traits. La variation d'un caractère chez un individu donné constitue son phénotype. C'est là, la première condition pour qu'il y ait sélection naturelle : au sein d'une population, certains caractères doivent présenter des variations, c'est le principe de variation.

II.2. Principe 2 : Les individus les plus adaptés au milieu survivent et se reproduisent davantage

Certains individus portent des variations qui leur permettent de se reproduire davantage que les autres, dans un environnement précis. On dit qu'ils disposent d'un avantage sélectif sur leurs congénères :

- La première possibilité est, par exemple, qu'en échappant mieux aux prédateurs, en étant moins malades, en accédant plus facilement à la nourriture, ces individus atteignent plus

facilement l'âge adulte, pour être apte à la reproduction. Ceux qui ont une meilleure capacité de survie pourront donc se reproduire davantage.

- Dans le cas particulier de la reproduction sexuée, les individus ayant survécu peuvent être porteurs d'un caractère particulièrement attirant pour les partenaires de sexe opposé. Ceux-là seront capables d'engendrer une plus grande descendance en copulant davantage.

Dans les deux cas, l'augmentation de la capacité à survivre et à se reproduire se traduit par une augmentation du taux de reproduction et donc par une descendance plus nombreuse, pour les individus porteurs de ces caractéristiques. On dit alors que ce trait de caractère donné offre un avantage sélectif, par rapport à d'autres. C'est dans ce principe d'adaptation uniquement, qu' intervient le milieu de

II.3. Principe 3 : Les caractéristiques avantageuses doivent être héréditaires

La troisième condition pour qu'il y ait sélection naturelle est que les caractéristiques des individus doivent être héréditaires, c'est-à-dire qu'elles puissent être transmises à leur descendance. En effet certains caractères, comme le bronzage ou la culture, ne dépendent pas du génotype, c'est-à-dire l'ensemble des gènes de l'individu. Lors de la reproduction, ce sont donc les gènes qui, transmis aux descendants, entraineront le passage de certains caracteres d'une generation a l' autre. C' est le principe d'hérédité.

Ces trois premiers principes entrainnent donc que les variations héréditaires qui confêrent un avantage sélectif seront davantage transmises à la génération suivante que les variations moins avantageuses. En effet les individus qui portent les variations avantageuses se reproduisent plus. Au fil des générations, on verra donc la fréquence des gènes désavantageux diminuer jusqu'à
éventuellement disparaître, tandis que les variations avantageuses se répandront dans la population, jusqu'à éventuellement être partagées par tous les membres de la population ou de l'espèce. Par exemple, dans la population humaine, la bipédie est un caractère commun à tous les êtres humains modernes. (Wikipédia)
\end{abstract}

Les exemples (5) et (6) illustrent un autre type de marqueurs d'items et font apparaître un autre aspect de la structure: sa capacité à organiser différentes dimensions du discours comme, par exemple, la dimension topicale, temporelle, rhétorique, événementielle ou argumentative. En (5), on énumère quatre intervalles temporels présentés comme des périodes par le syntagme numéral placé en titre. Le lecteur interprète le parallélisme entre les quatre syntagmes prépositionnels détachés en tête de paragraphe comme des marqueurs signalant chacune des périodes annoncées. Ces marqueurs réalisent donc conjointement les métafonctions textuelle et idéationnelle. C'est parce qu'ils sont mis visuellement sur le même plan et qu'ils sont syntaxiquement parallèles que les items forment un tout auquel le titre donne un sens. Dans cet exemple, on voit bien que la disposition - où chacun des « cadratifs » (Charolles et PéryWoodley, 2005) initie un segment qui se distingue du suivant par un changement de paragraphe constitue un élément du marquage.

\title{
(5) Quatre périodes
}

Entre 1949 et 1970, la production pétrolière américaine (brut et " condensats ") est multipliée par deux ; dans le même temps, la part de la demande couverte par le pétrole importé passe de $10 \%$ à $23 \%$. La croissance de la production intérieure atteste que le coût de renouvellement du " stock ", c'est-àdire des réserves, était compatible avec le prix en vigueur à l'époque. Toutefois, sur l'ensemble de cette période, la dépense nécessaire à l'ajout d'un baril de réserves au Moyen-Orient représente une petite fraction de celle requise aux États-Unis, et cette fraction diminue. Le pétrole du Moyen-Orient (mais aussi du Venezuela, et d'ailleurs) exerce donc, à partir des années cinquante, une pression A partir de 1970, toutes les formes d'investissement susceptibles d'augmenter les réserves de pétrole connaissent, aux États-Unis, des coûts fortement croissants. Les évènements de 1973 introduisent de nouveaux paramètres, en particulier réglementaires. L'explosion des prix du brut aurait dû favoriser un relatif redressement de la production intérieure et une baisse de la demande, donc une décroissance des importations. Mais les dispositions législatives prises pour soulager les raffineurs face à l'augmentation de leurs coûts d'approvisionnement (entitlements system) fonctionnent comme une subvention aux importations. Combinée à la réglementat
signal prix et distordent les incitations : le développement pétrolier intérieur est ralenti, la demande est artificiellement soutenue.

Entre 1978 et 1985, deux effets se conjuguent pour précipiter une chute des importations (Figure 10, Figure 11) :

- La compétitivité marginale de la production intérieure se redresse. La forte augmentation du prix mondial déclenche un véritable " boom " des investissements d'exploration et développement (dont les résultats sont décevants dans les 48 États " continentaux "). Elle permet en revanche une rapide et forte montée en puissance du champ géant de Prudhoe Bay en Alaska, découvert en 1967. Le développement de ce champ était rentable dès avant le choc pétrolier, mais il fut retardé jusqu'en 1973 par la bataille politique autour de la construction du pipeline trans-Alaska. D'autre part, la libéralisation des prix pétroliers en 1981 supprime les aides aux puits 198 .
des entitlements : entre 1981 et 1985 , même la production des lower 48 se redresse aux dépens des importations.

- L'ajustement de la demande, longtemps entravé par la réglementation des prix, s'effectue brutalement (-2 Mb/j entre 1979 et 1983$)$.

En conséquence, les importations chutent sur cette période, tant en valeur absolue $(-3,8 \mathrm{Mb} / \mathrm{j})$ que relative (-16 points de part de marché).

De 1985 à aujourd'hui, la part du pétrole importé dans la couverture de la demande ne cesse d'augmenter. La production américaine baisse au rythme de $2 \%$ par an en moyenne. Cette baisse ralentit après 1990, grâce notamment à la forte progression de l'offshore dans le Golfe du Mexique, stimulée par des mesures fiscales et par les progrès technologiques (cf. infra). Les importations ont progressé de plus de $5 \%$ par an en moyenne sur 15 ans, pour atteindre leur maximum historique en 2000 . Elles s'élèvent alors à $11 \mathrm{Mb} / \mathrm{j}$, soit $54 \%$ des besoins de l'économie et de la société américaines (Figure 10). (Géopolitique)

Dans l'exemple (6), on retrouve le même type de marqueur d'items qu'en (5). A ces syntagmes prépositionnels détachés, s'ajoute toutefois ici une série de syntagmes nominaux sujets. Dans la phrase qui précède la série d'items, cette double organisation est justifiée par les scientifiques français d'une part et très tôt d'autre part.

(6) Les scientifiques français s'intéressent à la cybernétique et à la théorie de l'information très tôt. En 1947, le mathématicien Szolem Mandelbrojt (1899 - 1983) invite Norbert Wiener (1894 - 1964) à un colloque à Nancy. Celui-ci se voit offrir la possibilité de publier en France un ouvrage sur le caractère unificateur de la cybernétique. C'est ainsi que Cybernetics fut publié en 1948 conjointement par les Editions Hermann à Paris et par les MIT Press et John Wiley \& En 1949, Léon Brillouin (1889 - 1969), membre de l'Ecole des Hautes études de New York depuis 1941, promeut la théorie de l'information en physique. En 1950, Louis de Broglie $(1892$ - 1987) organise une série de conférences ayant pour titre "Cybernétique. Théorie du signal et de l'information"qui conduisit à la reconnaissance de la théorie de l'information comme science autonome. 
Dans sa thèse publiée en 1953, Marcel-Paul Schützenberger (1920 - 1996) insiste sur le caractère unificateur pour les sciences de la théorie de l'information. (Linguistique)

Ces deux derniers exemples donnent à voir comment les items d'une série peuvent être clairement signalés en l'absence de MIL (voir également les titres de l'exemple (4)). Les SP cadratifs sont à même de jouer ce rôle, comme on l'a montré (5), où le titre joue un rôle décisif dans l'interprétation énumérative des quatre paragraphes suivants qui commencent tous par un SP cadratif fixant une période. En (6), en revanche, où l'amorce est moins explicite (cf. section suivante), la lecture énumérative de l'ensemble semble toutefois s'imposer grâce au strict parallélisme qui caractérise les phrases qui suivent. Toutes se conforment en effet à un même schéma : un SP cadratif temporel suivi d'un SN sujet dont la tête est un nom propre de mathématicien français. La structure de type chiasmatique utilisée ici nous semble justifier notre interprétation en termes de structure énumérative de l'ensemble du segment. Le chiasme contribue en effet à tisser le lien entre les deux composants annoncés dans l'amorce - les noms scientifiques français et les dates d'événements montrant leur intérêt pour la cybernétique et la théorie de l'information - qui se trouvent entrecroisés dans les items.

Par ailleurs, les exemples (4) et (5) illustrent une autre propriété remarquable des structures énumératives : la possibilité d'un enchâssement des structures, dit autrement l'un des items de la liste est l'amorce d'une autre liste (par exemple en (5), l'item-amorce annonce deux effets). Cette propriété s'ajoute à celles que nous avons illustrées ici et qu'on peut résumer ainsi : diversité des moyens utilisés pour signaler les items et caractère multi-échelle de la structure.

\section{Formes et fonctions des amorces et des clôtures}

Les exemples que nous venons d'examiner dans la section précédente montrent que l'interprétation énumérative d'un segment textuel est déclenchée par une pluralité d'éléments. Ces indices opèrent quelquefois seuls mais le plus souvent l'interprétation s'appuie sur une combinaison d'entre eux. On a vu comment, par exemple en (6), la répétition d'un même schéma associée au contenu sémantique de l'amorce fait apparaître la structure énumérative aux yeux du lecteur. C'est ce type d'observation qui nous conduit à défendre une conception de la structure énumérative comme un tout fonctionnel. Nous poursuivrons cette démonstration ici en suivant deux directions : vers l'amont, en examinant la diversité des réalisations de l'amorce, et vers l'aval, en observant la clôture, le segment textuel qui suit la série d'items.

Rappelons avant de passer à l'analyse ce qui distingue notre approche des données de celle des travaux antérieurs : comme nous l'avons dit plus haut, nous exploitons un vaste corpus de structures énumératives annotées dans des types de texte et des domaines diversifiés, à même de refléter la diversité des réalisations; de surcroît, ces annotations ont été produites sur la base de l'identification globale des structures et non de la présence de certains marqueurs.

\subsection{L'amorce}

Comme nous l'indiquions dans la section 2, les travaux auxquels nous pouvons nous référer ont tendu à se focaliser sur l'énumération - ainsi que le dénote le terme " série linéaire » - et en particulier sur les marqueurs d'items, délaissant l'amorce. Il existe toutefois des travaux s'intéressant au syntagme nominal susceptible d'exprimer le critère de co-énumérabilité : « classifieur cataphorique », " nom énumérable », selon les auteurs, qui envisagent pour certains la relation que ce nom entretient avec les items comme une relation d'hypo/hyperonymie exploitable automatiquement (Jacquemin et Bush, 2000 et plus récemment Fauconnier, 2013). Si les auteurs peuvent être amenés à proposer une modélisation des formes prises par ce syntagme nominal, il s'agit surtout pour eux de représenter les cas prototypiques (ou perçus comme tels), alors que notre approche en corpus annoté nous conduira au contraire à rendre compte de la complexité et à décrire un éventail bien plus étendu de réalisations. D’autres travaux, dans une perspective pragmatique, envisagent l'amorce comme un contrat qui engage le scripteur envers le lecteur. Nous retiendrons les propositions de Tadros (1994), à qui nous devons également le terme cité plus haut 
de «nom énumérable », et qui décrit l'amorce comme un élément "prédictif » qui signale que le scripteur s'engage contractuellement à énumérer.

L'importance de l'amorce dans l'économie d'ensemble de la structure énumérative est rendue évidente par sa présence dans $95 \%$ des structures annotées ${ }^{8}$. La réalisation des amorces témoigne toutefois d'une grande diversité et ce, à la fois en termes de fonction et de présence de marques identifiables. Tentant de cartographier cette diversité, la figure 1 propose, à partir de la notion de prédiction, de distinguer deux grands types d'amorce: d'une part, des amorces prédictives, que l'on pourrait aussi appeler contractuelles, comportant des traits de réalisation qui engagent le scripteur en déclenchant chez le lecteur une attente forte d'énumération; d'autre part, des amorces-annonces, dont cet aspect prédictif est absent, mais qui expriment ou permettent d'inférer - éventuellement a posteriori - le critère sous-jacent à la mise en parallèle des items, et dont la réalisation est très variable. Certains traits que nous identifions par les lettres $[\mathrm{S}],[\mathrm{P}],[\mathrm{N}]$ et $[\mathrm{T}]$ semblent peser fortement dans le déclenchement d'une attente d'énumération : ainsi, une amorce « syntaxiquement incomplète » (cf. Luc et al., 2000) où une place syntaxique attend d'être remplie par les items ([S]), une ponctuation finale par deux points ([P]), un syntagme nominal du type les (quatre) facteurs suivants $([\mathrm{N}])$. Ce syntagme nominal, qui exprime le critère de coénumérabilité, nécessiterait une description fine. En effet, certaines réalisations - c'est le cas de les quatre facteurs suivant-, semblent pouvoir à elles-seules déclencher une attente d'énumération, alors que d'autres vont devoir s'associer à d'autres traits pour produire une amorce prédictive. Quant au trait typodispositionnel alinéa ([T]), il a des fonctions multiples et ne peut que renforcer d'autres traits. Le modèle présenté en figure 1 devra certes être affiné, mais il servira ici à organiser une première analyse d'exemples correspondant à différentes configurations de traits.

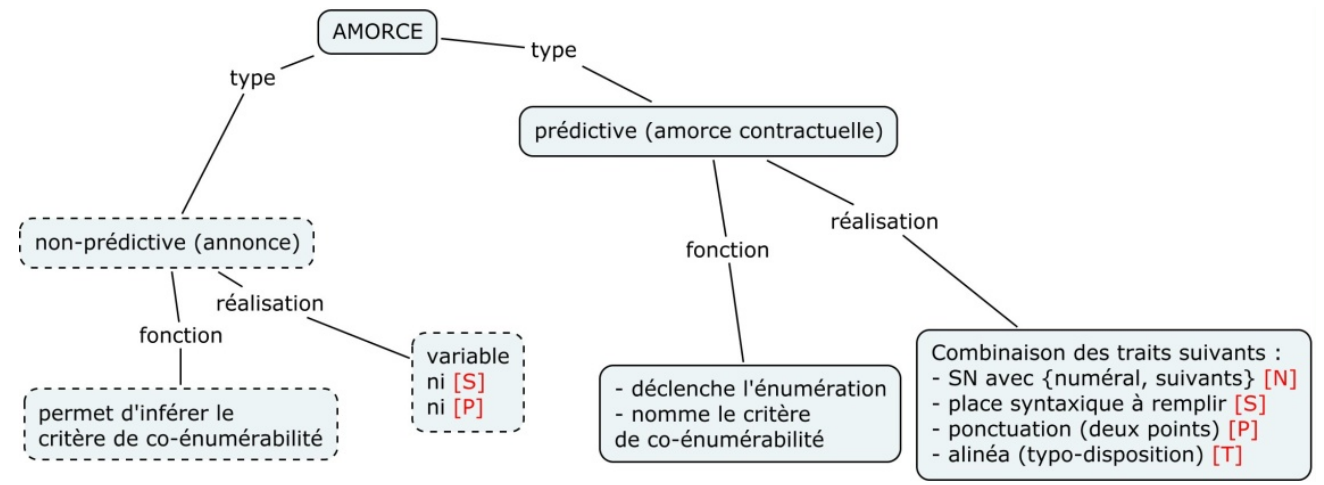

Figure 1 : Amorces prédictives et non-prédictives : fonctions et réalisations

Configuration 1: [NSPT] - La présence conjointe dans l'exemple (7) d'un SN avec déterminant numéral, d'une incomplétude syntaxique, des deux points et du passage à la ligne donne à voir comment l'absence d'une énumération à la suite créerait le trouble et serait une rupture de contrat entre scripteur et lecteur. L'exemple (8) partage les mêmes traits, mais la détermination indéfinie plurielle (plusieurs) semble moins contraignante qu'un numéral.

(7) Il prévoit une attaque sur trois axes, avec du nord au sud : (Wikipédia)

(8) Cette capacité dépend de plusieurs facteurs parmi lesquels : (Géopolitique)

Configuration 2: [NPT] - Cette configuration comporte le trait ponctuationnel et le trait typodispositionnel, qui contribuent à assurer une lecture prédictive même dans (11), bien que le SN D'autres motifs y appelle moins nettement une énumération qu'en (9) et (10). L'exemple (12) va à l'encontre de la conception selon laquelle que le SN de l'amorce devrait être au pluriel : un équilibre se décline sans difficulté dans la suite en deux items.

(9/cf. 22) $)^{9}$ L'approche de la LEC est, selon nous, particulièrement appropriée pour servir au développement d'une modélisation formelle et à un transfert des savoirs lexicographiques pour les raisons suivantes: (Linguistique) 
(10/ cf. 20) Dans un discours, Jaurès évoque la longue liste des faux qui parsèment le dossier Dreyfus, et insiste particulièrement sur deux pièces saillantes : (Wikipédia)

(11/ cf. 18) formulation moins nette du SN mais renfort de $\mathrm{P}$ et $\mathrm{T}$ D'autres motifs, cependant, viennent à l'esprit : (Linguistique)

(12) Pour tenter de concilier ces positions antagonistes, un équilibre délicat a été bâti à Doha : (Géopolitique)

Configuration 3 : [SPT] - L'exemple (13) présente un cas d'absence de SN auquel accrocher le critère de co-énumérabilité. La phrase se lit néanmoins comme une amorce prédictive en raison de la présence des autres traits. On verra en section 4.2 comment la clôture compense cette absence d'expression du critère de co-énumérabilité dans l'amorce.

\section{(13/ cf. 2) En substance, il est reproché à l'analogie : (Linguistique)}

Tous les exemples qui suivent sont caractérisés par l'absence des traits [S] (incomplétude syntaxique) et [P] (deux points). Selon la figure 1, cette absence les fait tomber dans la catégorie amorce non-prédictive. Il semble bien en effet y avoir une rupture à ce stade, même si la lecture prédictive ou non de l'amorce dépend alors surtout du SN dénotant le critère de co-énumérabilité. Ainsi, si l'on compare les exemples (14) et (15), qui sont des titres de section, l'attente d'énumération est sans doute plus nette pour (14) en raison de la présence d'un déterminant numéral. Mais, globalement, pour ces deux exemples, il y a lieu de se demander s'il n'y a pas retournement et si l'identification de l'amorce ne dépend pas de la perception de l'énumération qui la suit plutôt que l'inverse. Ce sera encore plus clair pour (16) et (17), qui ne possèdent aucun des traits de réalisation identifiés.

(14/ cf. 5) Quatre périodes (Géopolitique)

(15/ cf. 4) Principes de la sélection naturelle (Wikipédia)

(16/ cf. 6) Les scientifiques français s'intéressent à la cybernétique et à la théorie de l'information très tôt. (Linguistique)

(17/ cf. 3) Pourtant, les attaques du 11 septembre et la psychose entretenue par les découvertes d'enveloppes contenant du bacille du charbon donnent le sentiment qu'un cap a été franchi. (Géopolitique)

Nous en arrivons alors à l'analyse suivante : certes, certaines amorces sont remarquables parce qu'elles engagent le scripteur sur la nature de la suite du texte et que le lecteur peut se sentir légitimement floué si aucune énumération ne suit ; mais il demeure que ce type d'amorce, peut-être souhaitable dans certaines situations $^{10}$, ne semble pas nécessaire pour qu'une structure énumérative soit repérable et donc fonctionnelle (cf. la définition donnée en 2: «le scripteur dispose du texte de manière que le lecteur reconnaisse à la fois la disposition et l'intention qui est derrière »). Si les structures dont sont issues les amorces (14) à (17) fonctionnent, c'est parce que sur l'ensemble de la structure les indices sont suffisants pour induire une lecture énumérative. Que ce soit dans le contexte du traitement cognitif des textes ou du traitement automatique, il semble légitime de les envisager comme des systèmes à l'intérieur desquels des processus de compensation s'instaurent, en fonction des types de marques, de leur poids, de sorte que, lorsqu'on atteint un certain seuil de signalisation totale, l'interprétation énumérative s'impose. Pour illustrer, revenons à l'exemple (17) en contexte (se reporter à (3) pour la structure intégrale) : cette phrase exempte de tout trait caractéristique est suivie de trois items qui sont quant à eux très marqués (cf. analyse en 3) et qui doivent même lui être rattachés syntaxiquement pour faire sens. L'incomplétude syntaxique, trait fort des amorces prédictives, se retrouve donc ici dans les items. Notons enfin que les amorces non prédictives fournissent cependant des éléments pour l'interprétation de l'énumération, et en particulier pour identifier ou inférer le critère de co-énumérabilité.

Avant de passer à l'examen de la clôture, nous souhaitons revenir rapidement sur trois formes particulières que peut prendre l'amorce. La première de ces formes, le titre-amorce, est illustrée par (4) et (5), qui présentent deux configurations distinctes : alors qu'en (4) les items sont des sous-sections titrées qui développent les principes annoncés par le titre de niveau supérieur, les items de l'exemple (5) sont des paragraphes indexés par des adverbiaux temporels nommant les quatre périodes du titre. L'exemple (4) illustre également la seconde forme particulière, une structure décrite par Porhiel (2007:131) comme 
suit: «la deuxième énumération se greffe sur la première structure énumérative, constituant ainsi le deuxième temps d'une structure énumérative à deux temps ». Enfin, certaines amorces ne précèdent pas directement l'énumération, comme dans l'exemple (21), où une phrase s'interpose entre ce qu'on reconnaît comme l'amorce (Cette dynamique ou impulsion fondamentale se sépare en 8 dynamiques) et le premier des huit items. C'est donc ici non plus seulement la composition de l'amorce, mais son insertion dans le texte qui connait de nombreuses variations.

\subsection{La clôture}

Tel qu'il est utilisé, en littérature ou en linguistique, dans les travaux de description des listes, le terme clôture désigne généralement le dernier item d'une série. A l'écrit, il est signalé par des marqueurs comme enfin qui sont décrits par exemple par Turco \& Coltier (1988:60) comme des MIL temporels marquant la clôture, c'est-à-dire le dernier item (dans la même série, d'abord et ensuite sont dits marqueurs d'ouverture et de relais), mais il recouvre aussi très souvent les points de suspension qui s'insèrent à la suite de la séquence d'items laissant paradoxalement la liste ouverte. A l'oral, on peut évoquer le rôle d'une forme comme tout ça, dont Johnsen (2011) décrit bien le fonctionnement paradoxal : tout en étant bien disposé à marquer la clôture d'une liste, tout ça suggère davantage.

Dans les structures énumératives que nous avons examinées, les items forment une liste fermée. La clôture constitue donc pour nous ici un segment qui vient à la suite du dernier item de la liste plutôt que de prendre place dans la série, en position de dernier item. Jackiewicz $(2005: 106)$ remarque le rôle de ce segment. Mais, son travail portant principalement sur la description des MIL, elle se contente de signaler que les énumérations se trouvent parfois « clôturées » par ce qu'elle appelle « une rétro-évaluation portant sur l'ensemble de la série ». Nous revenons sur ce terme plus bas.

Il faut bien reconnaitre, avec Jackiewicz, que ce segment est relativement rare. Pour preuve, dans le vaste corpus annoté que nous avons exploité, seulement 131 segments ont été considérés comme des clôtures - ce qui représente moins de $15 \%$ de l'ensemble des structures annotées ${ }^{11}$. Notre intérêt pour ce segment conclusif se justifie cependant par le fait qu'il s'agit d'un lieu particulièrement intéressant pour observer le « devenir textuel » (terme que nous reprenons à Schnedecker, $2006: 267$ ) de la structure énumérative.

Si l'amorce s'avère un élément essentiel de la structure énumérative, la clôture ne l'est pas. Cela n'a toutefois rien d'étonnant, cette structure ayant en premier lieu une fonction de catégorisation. Qu'elles soient créées par le discours, comme dans l'exemple ci-dessous où l'auteur énumère une liste de motifs, ou qu'elles énumèrent des « objets du monde », les catégories ne sont pas nécessairement destinées à être reprises dans le texte.

(18) D'autres motifs, cependant, viennent à l'esprit :

(i) L'identité des ouvrages était-elle aisément repérable ? Si Le Petit Robert des enfants semble un nom bien choisi connotant le titre phare de la marque tout en nommant son public cible, Le Robert oral-écrit ne laissait pas présager de façon transparente l'usage d'un répertoire dont les usagers ne ressortaient pas non plus de façon tranchée, et Le Robert méthodique comme Le Robert brio sont deux dénominations opaques po

(ii) La complexité des dispositifs peut aussi être invoquée. Pour le Robert oral-écrit, elle était rédhibitoire, la difficulté inhérente à la consultation d'une nomenclature en alphabet phonétique (compensée il est vrai par un index des formes graphiques) étant accrue par le recours à des conventions de notation spécifiques : un projet de dictionnaire de cet ordre ne serait probablement viable que dans un espace multimédia dans lequel les consultants accéderaient aux articles en énonçant des formes lexicales. Le Robert méthodique, lui, a pu être perçu comme trop compliqué à utiliser, tant par les va-et-vient entre les articles consacrés à des mots complexes et ceux qui décrivaient leurs éléments de formation que par la signalétique des
découpages et des renvois, et certains des segrnents isolés par la procédure distributionnelle n'auront sans doute pas manqué de paraître contre-intuitifs à des utilisateurs pouvant également être découpages et des renvois, et certains des segments isolés par la procédure distributionnelle n'auront sans doute pas manqué de paraître contre-intuitifs à des utilisateurs pouvant également être
troublés par la mise à l'écart de l'approche étymologique, qui, à défaut d'être nécessairement plus lumineuse, est au moins plus familière. Quant au Petit Robert des enfants, son option troublés par la mise à l'écart de l'approche étymologique, qui, à défaut d'être nécessairement plus lumineuse, est au moins plus familière. Quant au Petit Robert des enfants, son option
d'éclatement modulaire des articles et la dissémination de leur texte dans trois colonnes, conçue comme un moyen de hiérarchiser clairement les informations en évitant la monotonie, peut avoir d'éć perçue à rebours comme une source de confusion. Enfin, conctions

(iii) Enfin, concernant ce dernier dictionnaire, on peut aussi se demander s'il n'a pas été desservi par un double déficit ergonomique eu égard à l'âge de ses destinataires : trop encombrant et lourd $(2 \mathrm{~kg})$ à manipuler et à transporter, avec des pages trop grandes et trop pleines comportant des lignes trop serrées.

(Linguistique)

On ne doit toutefois pas en conclure que la fonction de catégorisation serait le privilège de l'amorce. L'exemple (2) de notre introduction montre que c'est parfois à la clôture que revient le rôle de fournir explicitement le critère de co-énumérabilité. Dans cet exemple, en effet, le nom tête du syntagme chacune de ces critiques justifie la réunion des items qui font l'objet de l'énumération tout en la nommant.

Cela étant dit, essayons maintenant de répondre à la question de savoir à quoi sert la clôture quand elle est là. L'examen de nos données nous conduit à distinguer dans ce qui suit deux cas de figure: dans le premier, en l'absence d'amorce, c'est la clôture qui nomme le critère de co-énumérabilité et ce faisant fait 
apparaître la structure; dans le second, le critère exprimé une première fois dans l'amorce fait l'objet d'une nouvelle mention dans la clôture.

\subsubsection{La clôture « révèle » la structure}

L'interprétation énumérative du long extrait donné en (19) est déclenchée par le syntagme nominal démonstratif (désormais SND) sujet de la première phrase du dernier paragraphe : ces exemples. Ce syntagme dont la référence est décrite par Cornish (2011) comme relevant de ce qu'il appelle la « deixis de discours $»^{12}$ ne renvoie pas à une simple entité référentielle ni à une proposition mais à un ensemble de propositions particulièrement long ici puisqu'il s'agit de plusieurs paragraphes. Le nom-tête de ce syntagme a une fonction résomptive décrite initialement par Conte (1996:1) comme :

"a cohesive device by which a noun phrase functions as a resumptive paraphrase for a preceding portion of a text. This portion of a text (or segment) may be of various length and complexity (a whole paragraph or just one sentence)."

Il faut ajouter que la lecture énumérative est confortée, d'une part, par la disposition du texte puisque chaque paragraphe décrit la situation d'un pays, ce dernier étant, à deux exceptions près, le sujet de la première phrase du paragraphe. D'autre part, par le parallélisme syntaxique des phrases se trouvant en fin de paragraphe (mis en valeur typographiquement dans l'exemple). Les deux exceptions ne compromettent toutefois pas la lecture énumérative de l'ensemble parce qu'elles ne constituent que l'un des facteurs qui participent ici au signalement de la structure, les autres facteurs étant : le SND de clôture, la disposition (chaque item fait l'objet d'un paragraphe), le SN sujet dont la tête est un nom propre pays et le syntagme verbal : sortir de la guerre ruiné (diversement instancié dans chaque item).

(19) L'Allemagne suivit dès le début des années 30 une politique différente des recettes de l'orthodoxie libérale dominante à l'époque. Sous la responsabilité financière de Herr Schacht elle se lance dans une politique d'investissement massif, au départ principalement avec des objectifs civils. Galbraith écrira dans son livre sur "la monnaie " que la politique allemande fut à cette époque une lance dans une politique dinvestissement massif, au départ principalement avec des objectifs civils. Galbraith écrira dans son livre sur "la monnaie "que la politique allemande fut à cette époque une
politique keynésienne complète avant l'heure. La doctrine de Keynes est en effet qu'il faut rétablir par une politique d'investissement public l'équilibre perdu entre épargne et investissement. C'est de

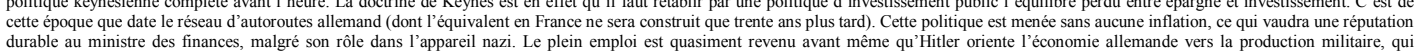
d'ailleurs, est largement réalisée ... en Union Soviétique pour contourner les traités. le Pacte Germano-Soviétique a été précédé par une longue et secrète coopération militaire. Il va de soi que l'Allemagne sortira de la guerre ruinée.

La situation est différente en Italie où l'exemple allemand n'est suivi que très partiellement et où les aventures coloniales extérieures absorbent une partie importante de l'énergie nationale. Elle sortira également de la guerre ruinée.

La France, principalement agricole, subit la crise de plein fouet, les exportations étant pratiquement arrêtées. Elle se replie sur son Empire. Les troubles sociaux et politiques qui aboutissent au Front populaire ne permettent pas l'élaboration d'une politique constante. Alfred Sauvy dans son " histoire économique de la France entre les deux guerres " constate que les " quarante heures " bloque la reprise qui commençait à se manifester. L'effort de production militaire est tardif et n'a qu'une influence marginale sur l'activité. La France sortira de la guerre pillée et ruinée.

La situation est peu ou prou la même au Royaume Uni qui a tenté de revenir à un taux de change en or intenable pour la Livre avant même 1929 et qui a connu une stagnation plus longue que les autres. La politique d'armement ne commence vraiment que très peu de temps avant la guerre et ne peut être considérée comme la méthode qui a permis de sortir de la crise. Elle sortira de la guerre victorieuse mais ruinée.

Le Japon connait une période d'avant guerre très différente des démocraties du fait de son expansionnisme militaire et de l'encadrement rigoureux de la population. Elle manque de pétrole pour ses entreprises. La guerre avec les Etats-Unis sera largement provoquée par l'embargo décidé par ce pays sur les exportations pétrolières vers le Japon. Le pays sortira ruiné par la guerre.

Les États-Unis connurent une période de forte activité pendant la guerre de quarante avec le retour au plein emploi, la mobilisation des hommes jeunes étant compensée par le recours massif à la main d'oeuvre féminine dans les usines d'armement. D'énormes investissements furent faits dans beaucoup de domaines qui donnèrent un avantage technologique au pays après guerre. Lorsque la guerre arriva à son terme, le retour des millions de soldats dans leurs foyers imposa une période de réajustement de l'économie. C'est cette transition qu'était censée faciliter le G.I. Bill. Au total ce fut le seul pays important à ne pas sortir ruiné de la guerre. La guerre avait également permis à des économistes keynésiens, sous l'influence de Hansen, de peupler l'administration qui se dote pendant la période des moyens en hommes, en idées et en droit, de son action. La paix retrouvée ils mirent en place une politique de dépense publique qui
ne se relâchera plus. ne se relâchera plus.

Ces exemples montrent que la montée vers la guerre ne sera nulle part le secret de la fin de la crise de 1929. La guerre marquera une rupture dans les mentalités, provoquera un besoin de reconstruction intense pendant une dizaine d'année, provoquera une concentration du pouvoir économique dans l'Etat qui est désormais partout chargé du droit au travail et à la sécurité sociale. Tous les gouvernements deviennent " keynésiens ". L'orthodoxie d'avant 1929 est morte. (Géopolotique)

\subsubsection{La clôture fournit une seconde mention du critère de co-énumérabilité}

Dans ce deuxième cas de figure, le critère exprimé une première fois dans l'amorce fait l'objet d'une nouvelle mention dans la clôture. On observe deux situations distinctes. La seconde mention peut faire appel à deux types de syntagmes : soit il s'agit d'un syntagme dont la tête est différente de celle qui a servi à introduire la liste d'items dans l'amorce; soit la tête de ce syntagme est identique à celle de celui qui a servi à fournir le critère de co-énumérabilité dans l'amorce. Nous examinerons successivement ces 
deux situations : la première sera illustrée par l'exemple (20) et la seconde par l'exemple (21). Il est remarquable de noter qu'il s'agit ici de deux formes privilégiées de réalisation de la clôture et qu'elles présentent des syntagmes nominaux démonstratifs (SND) dont la référence est à la fois anaphorique et déictique, autrement dit « anadéictique » (cf. Cornish (2011)). Et ce qu'il s'agisse, en suivant la typologie de Cornish, d' « anadeixis 'stricte' ${ }^{13} »$ (ces dynamiques, dans l'exemple 21) ou de « deixis de discours ${ }^{14} »$, en (1) avec ces critiques, en (4), avec ces exemples ou en (20) avec ces nouveaux faits :

(20) Dans un discours, Jaurès évoque la longue liste des faux qui parsèment le dossier Dreyfus, et insiste particulièrement sur deux pièces saillantes :

- La lettre de démission du général de Pellieux, rédigée en termes très durs. Juridiquement, elle a les formes d'un aveu de la collusion de l'État-Major: « Dupe de gens sans honneur, ne pouvant plus compter sur la confiance des subordonnés sans laquelle le commandement est impossible, et de mon côté, ne pouvant avoir confiance en ceux de mes chefs qui m'ont fait travailler sur des faux, je demande ma mise à la retraite.»

- Le bordereau prétendument annoté (par l'empereur Guillaume II) auquel le général Mercier avait fait allusion au procès de Rennes, et dont le fait rapporté par la presse aurait influencé les juges du Conseil de guerre.

Devant ces faits nouveaux, le général André, nouveau ministre de la Guerre, mène une enquête à l'instigation d'Émile Combes, assisté de magistrats. L'enquête est menée par le capitaine Targe, officier d'ordonnance du ministre. À l'occasion de perquisitions à la Section de statistiques, il découvre de très nombreuses pièces dont la majorité sont visiblement fabriquées. En novembre 1903, un rapport est remis au garde des Sceaux par le ministre de la Guerre. C'était le respect des règles, dès lors que le ministre constate une erreur commise en Conseil de guerre. C'est le début d'une nouvelle révision, avec une enquête minutieuse qui s'étend sur deux ans. (Wikipédia)

En (20), le segment qui suit la liste d'items contient un syntagme nominal qui a pour tête un nom différent de celui qui a servi à désigner le critère co-énumérabilité dans l'amorce : on est passé de pièce à fait. Ce nouveau $\mathrm{N}$ opère une reclassification des items de l'énumération introduits dans l'amorce par un nom concret (pièce) dans une catégorie abstraite (fait). Dit autrement, on passe d'un nom qu'on peut considèrer comme un hyperonyme à une caractérisation subjective de la part du scripteur qui fait le choix de désigner le contenu des propositions qui précèdent au moyen de ce que Schmid (2000) qualifie de « shell noun» et, plus précisément ici, d'un «modal shell noun » (fait appartient à la sous classe des « shells » dits « épistémiques de certitude »). A la suite de Conte (op. cit.) ou de Francis (1994), le terme de «shell noun », proposé par Schmid, vise à rendre compte de la capacité qu'ont ces noms à créer une sorte de « capsule » (ou de coquille) conceptuelle prête à accueillir un contenu informationnel complexe exprimé par une ou plusieurs propositions. Le scripteur utilise le nom fait pour fournir une caractérisation plus générale des deux items précédents et résumer au moyen d'une expression nouvelle (jamais apparue auparavant dans le texte) le contenu sémantique des propositions contenues ici dans les items de l'énumération. C'est ce que Schmid (op.cit. : 242) décrit comme une manière pour le scripteur de : "leave their mark on the conceptual content they shell" (probablement ce dont Jackiewicz a l'intuition quand elle utilise le terme de « rétro-évaluation »).

La fonction de ce nouveau référent dont la référence relève de la deixis de discours (comme dans nos deux exemples précédents) est de faire le lien entre le discours précédent et ce qui suit. On notera qu'il s'agit d'un référent qui n'est pas persistant puisqu'il disparaît aussitôt du discours. Ce type de nom contribue également très nettement à faire exister la structure dans son ensemble, comme un tout.

Notre second exemple illustre une situation particulièrement fréquente (cf. exemples (1) et (4)) où l'on retrouve en clôture le même nom que celui qui a été utilisé dans l'amorce :

(21) La scientologie considère que la motivation fondamentale de la vie est la survie, elle-même étant située sur une échelle graduée allant de la mort à l'immortalité potentielle. Cette motivation est appelée la dynamique. Cette dynamique ou impulsion fondamentale se sépare en 8 dynamiques. L'homme aurait une impulsion à survivre sur chacune d'entre elles.

- $\quad$ La première dynamique serait l'impulsion à survivre en tant qu'individu.

- La seconde dynamique l'impulsion à survivre à travers le sexe, la procréation et la famille.

- La troisième dynamique l'impulsion à survivre en tant que groupe ou en tant que groupes.

- La quatrième l'impulsion à survivre en tant qu'espèce; en l'occurrence l'humanité.

- La cinquième est l'impulsion à faire survivre la totalité des formes de vie : plantes, animaux, insectes, etc.

- La sixième est l'impulsion à survivre en tant qu'univers matériel (les énergies, la matière, les rochers, les planètes font partie de cette impulsion).

- La septième dynamique est l'impulsion à survivre en tant qu'esprits (la scientologie considère que la personne est un esprit « habitant un corps » et n'est ni son corps ni son mental).

- La huitième dynamique étant la dynamique de l'être suprême.

Toutes ces dynamiques sont des divisions arbitraires de la dynamique fondamentale qui les englobe toutes. (Wikipédia) 
Après avoir été introduit dans l'amorce via le syntagme nominal numéral : 8 dynamiques, le référent est repris dans la clôture par le même nom au sein d'un syntagme démonstratif accompagné d'un prédéterminant: Toutes ces dynamiques, dont la référence est strictement anadéictique (cf. " anadeixis 'stricte' »). Le démonstratif s'impose ici pour réactiver le référent, qui tout en étant toujours présent dans la mémoire discursive, n'est plus en position de focus une fois énumérées les huit dynamiques. Le SND fait donc plus que simplement maintenir la continuité référentielle avec le SN de première mention dans l'amorce puisqu'il s'est enrichi du contenu des items. Ce syntagme fait nettement apparaître la structure dans son ensemble dans la mesure où il englobe le contenu de l'amorce et des items. La référence anadéictique a ici une importante fonction stratégique à l'intérieur de la structure énumérative. Cette fonction consiste, tout en maintenant le lien avec ce qui précède et qui forme ainsi un tout, à signaler le début d'une nouvelle unité de discours.

Finalement, en dehors des rares cas où les clôtures nomment le critère de co-énumérabilité en l'absence d'amorce, leur fonctionnement s'explique par la conjonction de deux fonctions : une fonction de liaison et une fonction d'encapsulation - associant regard vers l'arrière et regard vers l'avant. Le regard vers l'arrière s'impose avec le SN démonstratif pour deux raisons. D'abord, c'est le SN démonstratif (ou un SN défini accompagné d'un marqueur de deixis textuelle comme « ci-dessus », cf. exemple (22)) qui est responsable de ce regard vers l'arrière et qui tient à la fois à la dimension déictique de ce syntagme et au type de nom utilisé. Il s'agit, comme on l'a dit, de noms abstraits dont la propriété est de résumer le contenu discursif essentiel de ce qui précède (c'est ce que Schmid (op. cit.), par exemple, appelle : "the signposting function of shell noun phrases"). La clôture est donc un segment qui prépare le lecteur à une transition entre la structure énumérative et l'unité de discours qui suit.

(22) L'approche de la LEC est, selon nous, particulièrement appropriée pour servir au développement d'une modélisation formelle et à un transfert des savoirs lexicographiques pour les raisons suivantes :

- $\quad$ elle est formelle (notions descriptives et formalismes de représentation précisément définis) et possède une valeur " universelle ", ayant été appliquée à une grande variété de langues très diverses ;

- $\quad$ elle vise spécifiquement la production de modèles lexicaux, appelés Dictionnaires explicatifs et combinatoires ou DEC (Mel'cuk et coll., 1984, 1988, 1992, 1999), et est appliquée à la construction de bases de données lexicales formelles, manipulables informatiquement - cf. la base de données DiCo du français (Polguère, 2000a ; Mel'cuk et Polguère, 2006) ou la base bilingue de collocations de Fontenelle (1997);

- $\quad$ elle sert aussi à la construction de modèles « vulgarisés » adaptés à l'enseignement et apprentissage de la langue - cf. le Lexique Actif du Français ou LAF (Polguère, 2000b ; Mel'cuk et Polguère, 2007) ou le dictionnaire en ligne DAFLES (Selva et coll., 2003)

- $\quad$ son potentiel d'application à la didactique des langues - déjà examiné dans le passé (Leed et Nakhimovsky, 1990 ; Steele, 1990 ; Gentilhomme, 1992) - a été confirmé par des études récentes (Tremblay, 2003) et intéresse de plus en plus les chercheurs en didactique ; elle est de plus utilisée avec succès dans d'autres domaines de linguistique appliquée, comme la terminologie (L'Homme, 2002).

L'énumération ci-dessus n'a pas pour finalité de démontrer que la LEC est la seule et unique approche disponible « sur le marché » pour mettre en oeuvre le transfert des savoirs lexicographiques. Elle vise simplement à justifier le caractère raisonnable de son utilisation pour atteindre les buts que nous nous fixons. (Linguistique)

\section{Conclusion}

Plutôt que de focaliser notre intérêt sur la description des moyens linguistiques utilisés pour signaler les items d'une énumération, comme l'avaient fait beaucoup de nos prédécesseurs, nous avons considéré la structure énumérative comme un tout fonctionnel. Cette conception de la structure nous a amenées à prendre en compte une diversité de traits participant à son signalement. Parmi eux, nous avons notamment proposé de compter les titres ou les adverbiaux en position initiale. Nous espérons avoir montré dans quelles conditions les uns et les autres contribuent à signaler une structure énumérative.

Telle que nous avons proposé de la décrire, la structure énumérative consiste a minima en une séquence d'items (l'énumération proprement dite) et l'expression d'un critère justifiant leur réunion au sein d'un même ensemble (le critère de co-énumérabilité). Qu'il soit explicite ou implicite, ce critère est à chercher dans le contexte qui précède (l'amorce) ou quelque fois dans celui qui suit (la clôture). Notre description s'est donné comme objectif de montrer comment s'agencent ces différents éléments et selon quelles configurations pour faire apparaître la structure aux yeux du lecteur. Une quantification précise de ces agencements et configurations serait nécessaire pour compléter cette description, quantification qui nécessiterait une vérification systématique de l'ensemble des annotations. 
Dans ces agencements, l'amorce joue un rôle décisif de prédiction, au sens où elle engage le scripteur à énumérer. Quand l'amorce n'engage pas à énumérer ce sont d'autres indices qui prennent le relais pour rendre saillante l'intention énumérative du scripteur. Quelle que soit la réalisation d'une structure énumérative - indices variés, potentiellement distribués sur les différents composants - elle se doit d'être perceptible, puisque c'est cette perception qui entraînera chez le lecteur la compréhension de l'intention qui la sous-tend. On pourrait modéliser ce fonctionnement par le biais d'indices pondérés qui s'additionnent (en fonction de contraintes à définir) jusqu'à atteindre un seuil à partir duquel la structure devient perceptible. La notion de rupture évoquée pour l'amorce (amorce prédictive $v s$ non-prédictive) pourrait ainsi être étendue à l'ensemble de la structure, avec l'idée d'un basculement dans l'interprétation une fois le seuil atteint.

La clôture et plus précisément le syntagme nominal démonstratif en position initiale du segment qui suit immédiatement la série d'items (qu'il assure la fonction sujet ou qu'il se trouve au sein d'un syntagme prépositionnel cadratif) a, quant à elle, une fonction de liaison. Ce syntagme démonstratif parce qu'il a à sa tête un nom abstrait qui encapsule le contenu de chacun des items - ainsi que le cas échéant la première mention de ce nom dans l'amorce - fait clairement apparaître la structure comme constituant une seule et même unité discursive.

Les noms qui servent à expliciter le critère de co-énumérabilité dans le corpus de structures énumératives que nous avons examiné sont généralement des noms abstraits. Dans les textes de notre corpus, qui inclut pourtant, rappelons-le, des articles de l'encyclopédie en ligne Wikipédia, l'énumération n'est qu'exceptionnellement utilisée pour fournir une liste de co-hyponymes - ce qui constitue potentiellement un obstacle aux travaux qui en TAL, à la suite de Jacquemin et Bush (2000), font l'hypothèse que les énumérations constituent un objet textuel pertinent pour l'acquisition lexicale. La vision de l'énumération qui prévaut dans ces travaux est celle de la liste telle que la définit Schiffrin (2006:163) :

"When making a list, a speaker brings together in one text a collection of items that can be classified as the same by some criteria, but different by others. The sameness of the items in a list justifies their inclusion in a single group (i.e. as a category (Rosch 1978)) and the differences between them separate them into subgroups (i.e. as subcategories)."

La liste est, en effet, l'instrument par excellence de la catégorisation: elle est le reflet de catégories préconstruites (les instruments à cordes, les rapaces prédateurs, les fruits et légumes à acheter au marché, etc.). Or, rares sont, dans notre corpus, les structures énumératives où l'amorce contient un hyperonyme dont les items sont les co-hyponymes. Mais comme le dit également Schiffrin :

"Although lists can present a set of taxonomic categories in which each entity is an example of the class through which it is known, they can also present more ad hoc collections (Barsalou, 1983) or schematic knowledge in which each entity is known through its participation or place in a collection (Mandler, 1984)."

Dans les structures énumératives utilisées dans les textes de notre corpus, on trouve en effet massivement ce que l'auteur appelle des catégorisations $a d$ hoc. Plus précisément, là où on aurait pu s'attendre à trouver des hyperonymes, des noms abstraits sont employés. On trouve par exemple : conséquences, faits, critiques, exemples, effets, critères, étapes, facteurs, motifs, idées, éléments, phénomènes.

Ce rapide examen suggère que, tout en étant la fonction première de la structure énumérative, la catégorisation ne sert pas uniquement à exprimer des classifications existantes. La diversité des exemples que nous avons examinés dans cette étude nous conduit à affirmer au contraire qu'elle est davantage utilisée pour «construire » des catégories pertinentes pour le discours du scripteur, ce qui fait d'elle un élément important du dispositif d'argumentation. Le rôle et le fonctionnement de la catégorisation nécessiterait d'être examiné plus en détail dans une nouvelle étude, consacrée cette fois à la valeur sémantique de l'énumération. 


\section{Références bibliographiques}

Bras, M., Prévot, L. et Vergez-Couret, M. (2008). Quelle(s) relation(s) de discours pour les structures énumératives ?, Actes du Colloque Mondial de Linguistique Française CMLF'08, J. Durand, B. Habert, B. Laks (éds.), 19451964, Paris, 9-12 juillet 2008.

Charolles, M. et Péry-Woodley, M-P. (2005). Les adverbiaux cadratifs. Langue Française, 148, 3-8.

Conte, M.-E. (1996). Anaphoric encapsulation. Belgian Journal of Linguistics, 10, 1-10.

Cornish, F. (2011). Strict anadeixis, discourse deixis and text structuring. Language Sciences, 33, 753-767.

Cornish, F (à paraître). Indexicals and L2 learners' metadiscursive awareness. In Sancho Guinda C., Breeze R. (eds), Creativity, Critical Thinking, Autonomy, and Motivation in English-Medium University Contexts. Berlin: Springer.

Fauconnier, J.-P., Kamel, M., Rothenburger, B. et Aussenac-Gilles, N. (2013). Apprentissage supervisé pour l'identification de relations sémantiques au sein de structures énumératives parallèles. Actes de la $20^{\mathrm{e}}$ conférence Traitement Automatique des Langues Naturelles (TALN 2013), Les Sables d'Olonne, France, 132-145.

Francis, G. (1994). Labelling Discourse: An Aspect of Nominal-Group Lexical Cohesion. In Coulthard M. (ed.), Advances in Written Text Analysis. London - New York: Routledge, 83-101.

Guillot, C. (2006). Démonstratif et déixis discursive : analyse comparée d'un corpus écrit de français médiéval et d'un corpus oral de français contemporain. Langue française, 152, 56-69.

Halliday, M.A.K. (1977). Text as semantic choice in social contexts. In van Dijk T. \& Petöfi J. (eds), Grammars and Descriptions. Berlin: Walter de Gruyter, 176-226.

Hempel, S. et Degand, L. (2008). Sequencers in Different Text Genres: Academic Writing, Journalese and Fiction. Journal of Pragmatics, 40 (4), 676-693.

Ho-Dac, L-M., Fabre, C., Péry-Woodley, M-P., Rebeyrolle, J., et Tanguy, L. (2012). An Empirical Approach to the Signalling of Enumerative Structures, Discours [En ligne], 10 2012.

Ho-Dac, L-M. et Péry-Woodley, M-P. (2014). Actes CMLF 2014, Berlin.

Jackiewicz, A. (2005). Les séries linéaires dans le discours. Langue française, 148, 95-110.

Jacquemin, C. et Bush, C. (2000). Fouille de Web pour la collecte d'entités nommées. Actes de la conférence Traitement Automatique des Langues Naturelles (TALN 2000), Lausanne, Suisse, 187-196.

Johnsen, L. A. (2011). Un éclairage sur le fonctionnement référentiel de tout ça en fin de liste. In Béguelin M.J., Corminboeuf G. (éds.), Du système linguistique aux actions langagières. Mélanges en l'honneur d'Alain Berrendonner. Bruxelles : De Boeck-Duculot, 487-505.

Luc, C., Mojahid, M., Péry-Woodley, M-P. et Virbel, J. (2000). Les énumérations : structures visuelles, syntaxiques et rhétoriques. In Gaio M. et Trupin É. (éds.), Document électronique dynamique : actes du troisième colloque international sur le document électronique (4-6 juillet 2000, Lyon). Paris : Europia, 21-40.

Maurel, F., Mojahid, M., Vigouroux, N., \& Virbel, J. (2006). Documents numériques et transmodalité. Transposition automatique à l'oral des structures visuelles des textes. Document numérique, 9 (1), 25-42.

Milcent-Lawson, S., Lecolle, M. et Michel, R. (sous la dir. de). (2013). Liste et effet liste en littérature. Classiques Garnier, collection « Rencontres ».

Péry-Woodley, M.-P., Afantenos, S. D., Ho-Dac, L.-M., et Asher, N. (2012). La ressource ANNODIS, un corpus enrichi d'annotations discursives. TAL, 52 (3), 71-101.

Porhiel, S. (2007). Les structures énumératives à deux temps. Revue Romane, 42 (1), 103-135.

Power, R., Scott, D. et Bouayad-Agha, N. (2003). Document Structure. Computational Linguistics, 29 (2), 211-260.

Schiffrin, D. (2006). In other words. Variation in reference and narrative. Cambridge: Cambridge University Press.

Schmid, H.J. (2000). English Abstract Nouns as Conceptual Shells: From Corpus to Cognition. Walter de Gruyter.

Schnedecker, C. (2006). De l'un à l'autre et réciproquement: aspects sémantiques, discursifs et cognitifs des pronoms anaphoriques corrélés (l'un / l'autre et le premier / le second). Bruxelles - Paris: De Boeck-Duculot. 
Tadros, A. (1994). Predictive Categories in Expository Texts. In Coulthard M. (ed.), Advances in Written Text Analysis. London - New York: Routledge, 69-82.

Turco, G. et Coltier, D. (1988). Des agents doubles de l'organisation textuelle, les marqueurs d'intégration linéaire. Pratiques, 57, 57-79.

\footnotetext{
${ }^{1}$ Pour la présentation des exemples, nous avons opté pour le compromis suivant : ne pas les couper mais jouer sur la taille de police pour en diminuer le volume. Les éléments importants pour notre exposé sont ainsi mis en valeur sans qu'on perde la perception de l'empan de texte concerné par la structure.

${ }^{2}$ La notion de «Marqueur d'Intégration Linéaire » (Auchlin, 1981)) est reprise par Turco \& Coltier (1988) qui en proposent un classement (numération, lieu, temps).

${ }^{3}$ Nous renvoyons le lecteur à Porhiel (2007) pour une revue assez complète et bien articulée des travaux sur les structures énumératives jusqu'à 2005 .

${ }^{4}$ Ressource disponible sur le site REDAC : http://redac.univ-tlse2.fr/, et décrite dans Péry-Woodley et al. (2011).

${ }^{5}$ Pour une description plus détaillée, nous renvoyons le lecteur à Ho-Dac et al. (2012). Les exemples étiquetés «Linguistique » sont issus des actes du premier Congrès Mondial de Linguistique Française ; ceux qui sont étiquetés « Géopolitique » proviennent d'articles produits par l'IFRI (Institut français des relations internationales), think-tank ou « laboratoire d'idées » français consacré à l'analyse des questions internationales; enfin, ceux qui sont suivis de la mention « Wikipédia » sont issus du sous-corpus constitué de 30 articles complets de Wikipédia.
}

${ }^{6}$ La ressource ANNODIS compte 991 structures énumératives annotées.

7 "Informally, document structure describes the organization of a document into graphical constituents like sections, paragraphs, sentences, bulleted lists, and figures; it also covers some features within sentences, including quotation and emphasis." (Power et al., $2003: 214$ ).

${ }^{8}$ Notons que ce pourcentage résulte d'une réannotation par nos soins, certaines amorces ayant échappé aux annotateurs lors de la création de la ressource: amorces non-adjacentes, titres-amorces. Une telle réannotation constitue un deuxième temps et non un désaveu du travail des annotateurs. Lors de cette réannotation, nous avons systématiquement examiné le texte en amont des structures annotées sans amorces.

${ }^{9}$ Pour les amorces issues d'exemples donnés in extenso ailleurs dans l'article, nous ajoutons au numéro d'exemple le numéro correspondant à cette présentation complète pour que le lecteur puisse s'y reporter.

${ }^{10}$ Nous pensons aux travaux de Maurel et al. sur l'oralisation des énumérations (cf. Maurel et al., 2006)).

${ }^{11}$ Contrairement à l'analyse des amorces, nous n'avons pas réannoté les structures annotées sans clôture (beaucoup plus nombreuses). Ce pourcentage ne prend donc pas en compte les clôtures ayant échappé à la vigilance des annotateurs.

${ }^{12}$ Dans d'autres travaux, on parle d'« anaphore résomptive» (cf. Guillot, 2006), pour désigner ces anaphores qui subsument le contenu du discours précédent grâce à la tête lexicale d'un SND. La condition d'une telle anaphore est que la tête du SN soit nouvelle, dit autrement qu'elle n'ait pas fait l'objet d'une première mention dans le co-texte précédent. A la suite de Cornish (2011), nous considèrerons qu'il n'y a pas anaphore ici mais que le syntagme ces exemples réalise la deixis de discours.

${ }^{13}$ Dans un article à paraître, Cornish définit l'anadeixis stricte de la manière suivante : "the subsequent reference to an entity which may have been evoked earlier in a discourse, but which is no longer - or is not yet - topical at the point of reference: the referent thereby targeted exists in the surrounding discourse, but is not readily accessible hence the involvement of the deictic dimension."

${ }^{14}$ Dans ce même article, Cornish décrit la deixis de discours comme : "the act of cognitive pointing towards a discourse representation in working memory, and the creation from it via an inference of a partly new discourse entity: the deictic dimension thus performs an even more dominant role in this type of indexical reference - hence its name." 